EPJ Web of Conferences 71, 00135 (2014)

DOI: 10.1051/epjconf/20147100135

(C) Owned by the authors, published by EDP Sciences, 2014

\title{
Non-relativistic particles in a thermal bath
}

\author{
Antonio Vairo ${ }^{a}$ \\ ${ }^{1}$ Physik-Department, Technische Universität München, \\ James-Franck-Str. 1, 85748 Garching, Germany
}

\begin{abstract}
Heavy particles are a window to new physics and new phenomena. Since the late eighties they are treated by means of effective field theories that fully exploit the symmetries and power counting typical of non-relativistic systems. More recently these effective field theories have been extended to describe non-relativistic particles propagating in a medium. After introducing some general features common to any non-relativistic effective field theory, we discuss two specific examples: heavy Majorana neutrinos colliding in a hot plasma of Standard Model particles in the early universe and quarkonia produced in heavy-ion collisions dissociating in a quark-gluon plasma.
\end{abstract}

\section{Introduction}

Heavy particles are a window to new physics for they may be more sensitive to new fundamental degrees of freedom. Some of these new degrees of freedom may be themselves heavy particles (like, for instance, the heavy neutrinos that we will discuss in section 2). Heavy particles can also be clean probes of new phenomena emerging in particularly complex environments. Examples are heavy quarks and quarkonia as probes of the state of matter formed in heavy-ion collisions.

We call a particle heavy if its mass $M$ is much larger than any other scale $E$ characterizing the system. The scale $E$ may include the spatial momentum of the heavy particle, masses of other particles, $\Lambda_{\mathrm{QCD}}$, symmetry breaking scales, the temperature $T$ of the medium and any other energy or momentum scale that describes the heavy particle and its environment. Under this condition the heavy particle is also non relativistic. The hierarchy $M \gg E$ calls for a low energy description of the system in terms of a suitable effective field theory (EFT) whose degrees of freedom are a field $H$ encoding the low-energy modes of the heavy particle, and all other low-energy fields of the system. In a reference frame where the heavy particle is at rest up to fluctuations that are much smaller than $M$, the EFT Lagrangian has the general form

$$
\mathcal{L}=H^{\dagger} i D_{0} H+\text { higher-dimension operators } \times \text { powers of } \frac{1}{M}+\mathcal{L}_{\text {light fields }} .
$$

In the heavy-particle sector the Lagrangian is organized as an expansion in $1 / M$. Contributions of higher-dimension operators to physical observables are counted in powers of $E / M$. It is crucial to note that the EFT Lagrangian can be computed setting $E=0$. Hence its expression is independent of the low-energy dynamics. The prototype of the EFT (1) is the heavy quark effective theory $[1,2]$.

a e-mail: antonio.vairo@ph.tum.de

This is an Open Access article distributed under the terms of the Creative Commons Attribution License 2.0, which permits unrestricted use, distribution, and reproduction in any medium, provided the original work is properly cited. 
In this contribution, we will concentrate on the case of a heavy particle of mass $M$ propagating in and interacting with a medium characterized by a temperature $T$ much smaller than $M$. This is a special case of the previous one. The EFT Lagrangian that describes the system at an energy scale much lower than $M$ has again the structure (1). As pointed out before, the temperature does not enter in the computation of the Lagrangian, which is fixed by matching at $T=0$. This means that the Wilson coefficients encoding the contributions of the high-energy modes can be computed in vacuum.

The temperature is introduced via the partition function of the EFT and affects the computation of the observables. Contributions of higher-dimension operators are counted in powers of $T / M$. In order to study the real-time evolution of physical observables and in particular decay widths, it is convenient to compute the partition function in the so-called real-time formalism. This consists in modifying the contour of the partition function to allow for real time (see e.g. [3]). In real time, the degrees of freedom double. However, in the heavy-particle sector the second degrees of freedom decouple from the physical degrees of freedom so that, as long as loop corrections to light particles can be neglected, the only difference with $T=0$ EFTs consists in the use of thermal propagators [4].

In the following, we will consider heavy particles interacting weakly with a weakly-coupled plasma. We will compute for them the corrections to the width induced by the medium, which we call their thermal width, $\Gamma$. In particular, in section 2 we will compute the thermal width of a heavy Majorana neutrino interacting weakly with a plasma of massless Standard Model (SM) particles in the primordial universe. Whereas in section 3 we will compute the thermal width of a quarkonium, which, like the $\Upsilon(1 S)$, is heavy enough to be considered a non-relativistic Coulombic bound state, and is produced in heavy-ion collisions of sufficient high energy that the formed medium is a weakly-coupled plasma of light quarks and gluons.

\section{Heavy Majorana neutrinos}

We consider a heavy Majorana neutrino, described by a field $\psi$ of mass $M$ much larger than the electroweak scale, $M \gg M_{W}$, coupled to the SM only through Higgs-lepton vertices:

$$
\mathcal{L}=\mathcal{L}_{\mathrm{SM}}+\frac{1}{2} \bar{\psi} i \not \phi \psi-\frac{M}{2} \bar{\psi} \psi-F_{f} \bar{L}_{f} \tilde{\phi} P_{R} \psi-F_{f}^{*} \bar{\psi} P_{L} \tilde{\phi}^{\dagger} L_{f},
$$

where $\mathcal{L}_{\mathrm{SM}}$ is the SM Lagrangian with unbroken $\mathrm{SU}(2)_{\mathrm{L}} \times \mathrm{U}(1)_{\mathrm{Y}}$ gauge symmetry, $\tilde{\phi}=i \sigma^{2} \phi^{*}$, with $\phi$ the Higgs doublet, $L_{f}$ are lepton doublets with flavor $f, F_{f}$ is a (complex) Yukawa coupling and $P_{L}=$ $\left(1-\gamma^{5}\right) / 2, P_{R}=\left(1+\gamma^{5}\right) / 2$ are the left-handed and right-handed projectors respectively. This extension of the SM provides a model for neutrino mass generation through the seesaw mechanism $[5,6]$. It also provides a model for baryogenesis through thermal leptogenesis [7, 8]. For a recent review see [9].

Let us consider baryogenesis. Differently from the SM, the model (2) has the potential to originate a large baryon asymmetry. The mechanism is the following. At a temperature $T \lesssim M$ the neutrino falls out of equilibrium. ${ }^{1}$ This happens because, as the temperature decreases, recombination processes become less and less frequent while the neutrino decays in the plasma. Since the neutrino is a Majorana particle, a net lepton asymmetry is generated. This is transferred to a baryon asymmetry through sphaleron transitions. The phases of the Yukawa couplings $F_{f}$ in the Lagrangian (2) provide extra sources of $\mathrm{C}$ and $\mathrm{CP}$ violations besides those in the $\mathrm{SM}$. Finally the generated baryon asymmetry is protected from washout after sphaleron freeze-out at a temperature $T \sim M_{W}$. Hence the model may fulfill the three necessary Sakharov conditions for baryon asymmetry [10] in a stronger way than the SM does and account for the observed baryon asymmetry in the universe.

\footnotetext{
${ }^{1}$ In (2) we have simplified the realistic case with more neutrino generations by considering only one heavy Majorana neutrino.
} 
ICNFP 2013

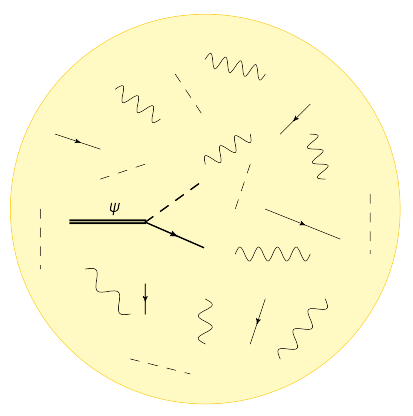

Figure 1. Heavy Majorana neutrino decaying in the early universe plasma.

An important quantity for leptogenesis is the rate at which the thermal plasma of the early universe creates Majorana neutrinos with mass $M$ at a temperature $T$. This quantity is in turn related to the heavy Majorana neutrino thermal width in the plasma, see figure 1. We consider the temperature regime $M \gg T \gg M_{W}$. The heavy Majorana neutrinos are non-relativistic, with momentum

$$
p^{\mu}=M v^{\mu}+k^{\mu}, \quad k^{\mu} \ll M .
$$

In kinetic equilibrium the residual momentum $k^{\mu}$ is of order $\sqrt{M T}$; far out of equilibrium it is of order $T$. If the neutrino is at rest up to fluctuations that are much smaller than $M$, then $v^{\mu}=(1, \mathbf{0})$.

At an energy scale much smaller than $M$ the low-energy modes of the Majorana neutrino are described by a field $N$ whose effective interactions with the SM particles are encoded in the EFT [11]: ${ }^{2}$

$$
\mathcal{L}=\mathcal{L}_{\mathrm{SM}}+\mathcal{L}_{\mathrm{N}}
$$

where

$$
\mathcal{L}_{\mathrm{N}}=\bar{N}\left(i \partial_{0}-\frac{i \Gamma_{T=0}}{2}\right) N+\frac{\mathcal{L}^{(1)}}{M}+\frac{\mathcal{L}^{(2)}}{M^{2}}+\frac{\mathcal{L}^{(3)}}{M^{3}}+O\left(\frac{1}{M^{4}}\right) .
$$

The Lagrangian (5) is of the type (1), the only difference being that the Majorana neutrino is a gauge singlet with a finite width at zero temperature, $\Gamma_{T=0}$, due to its decay into a Higgs and lepton.
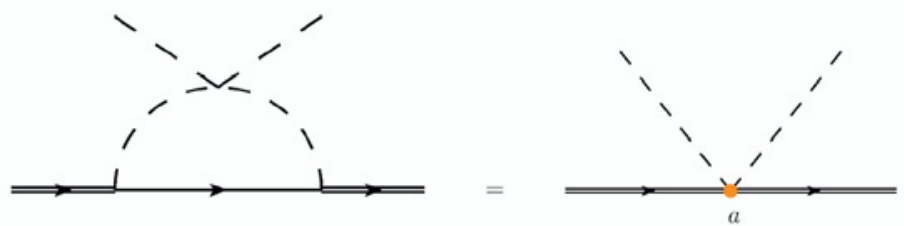

Figure 2. One-loop matching condition for the neutrino-Higgs coupling.

The power counting of the EFT indicates that the leading operators responsible for the neutrino thermal decay are dimension 5 operators contributing to $\mathcal{L}^{(1)}$. The symmetries of the EFT allow only for one possible dimension 5 operator, which is

$$
\mathcal{L}^{(1)}=a \bar{N} N \phi^{\dagger} \phi .
$$

This describes the scattering of Majorana neutrinos with Higgs particles. Scatterings of Majorana neutrinos with gauge bosons, leptons or quarks are subleading. The Wilson coefficient $a$ is fixed at one loop by the matching condition shown in figure 2 . The left-hand side stands for an (in-vacuum)

\footnotetext{
${ }^{2}$ An EFT for heavy Majorana fermions has been considered also in [12].
} 
diagram in the fundamental theory (2), whereas the right-hand side for an (in-vacuum) diagram in the EFT. Double lines are neutrino propagators, single lines lepton propagators and dashed lines Higgs propagators. For the decay width only the imaginary part is relevant; it reads

$$
\operatorname{Im} a=-\frac{3}{8 \pi}|F|^{2} \lambda
$$

where $\lambda$ is the four-Higgs coupling.

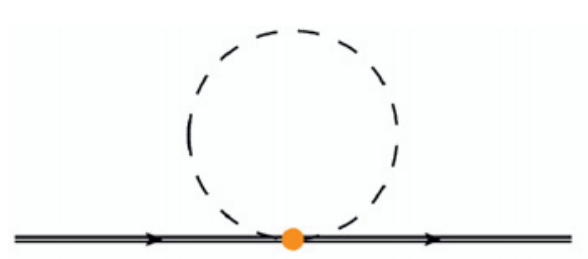

Figure 3. Higgs tadpole contributing to the neutrino thermal width.

The thermal width induced by (6) may be computed from the tadpole diagram shown in figure 3, where the dashed line has to be understood now as a Higgs thermal propagator. The leading thermal width reads [13, 14], [11], [15]

$$
\Gamma=2 \frac{\operatorname{Im} a}{M}\left\langle\phi^{\dagger}(0) \phi(0)\right\rangle_{T}=-\frac{|F|^{2} M}{8 \pi} \lambda\left(\frac{T}{M}\right)^{2},
$$

where $\left\langle\phi^{\dagger}(0) \phi(0)\right\rangle_{T}$ stands for the thermal condensate of the field $\phi$. Note that in the EFT the calculation has split into a one-loop matching, shown in figure 2 , which can be done in vacuum, and the calculation of a one-loop tadpole diagram, shown in figure 3, which is done in thermal field theory. The resulting simplification of the calculation with respect to a fully relativistic treatment in thermal field theory is typical of the EFT approach.

In a similar fashion one can calculate $T / M$ suppressed corrections to the thermal decay width. Also for them the calculation splits into an in-vacuum matching of higher-dimension operators in the expansion (5) and in the calculation of one-loop tadpole diagrams in thermal field theory. Only dimension 7 operators contribute to the width at next order in $T / M$. These are eight operators belonging to $\mathcal{L}^{(3)}$. Two each describe couplings of the Majorana neutrino to Higgs particles, leptons, quarks and gauge bosons respectively. Finally, the thermal width at first order in the SM couplings and at order $T^{4} / M^{3}$ reads [14], [11]

$$
\Gamma=\frac{|F|^{2} M}{8 \pi}\left[-\lambda\left(\frac{T}{M}\right)^{2}+\frac{\lambda}{2} \frac{\mathbf{k}^{2} T^{2}}{M^{4}}-\frac{\pi^{2}}{80}\left(3 g^{2}+g^{\prime 2}\right)\left(\frac{T}{M}\right)^{4}-\frac{7 \pi^{2}}{60}\left|\lambda_{t}\right|^{2}\left(\frac{T}{M}\right)^{4}\right],
$$

where $g$ is the $\mathrm{SU}(2)$ coupling, $g^{\prime}$ the $\mathrm{U}(1)$ coupling, and $\lambda_{t}$ the top Yukawa coupling.

\section{Heavy quarkonia}

Heavy quarkonia are bound states of heavy quarks. A quark is considered heavy if its mass $M$ is much larger than the typical hadronic scale $\Lambda_{\mathrm{QCD}}$. Quarkonia include bound states of charm and bottom quarks. They are a probe of the state of matter made of gluons and light quarks formed in high-energy heavy-ion collisions [16]. The reasons are that heavy quarks are formed early in heavyion collisions, $1 / M \sim 0.1 \mathrm{fm} \ll 1 \mathrm{fm}$, hence heavy quarkonium formation is sensitive to the medium, and that the quarkonium dilepton decay provides a clean experimental signal. The dissociation of a heavy quarkonium in a plasma of quarks and gluons is sketched in figure 4 . 


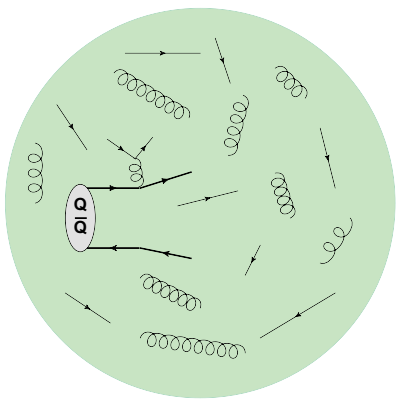

Figure 4. Heavy quarkonium dissociating in a plasma of light quarks and gluons.

Under the condition $M \gg T$ and for quarkonia formed almost at rest in the laboratory rest frame, the mass $M$ of the heavy quark is the largest scale in the system; as in the general framework discussed in the introduction, we may consider quarkonia non-relativistic particles suitable to be described by non-relativistic EFTs of the type (1). Differently from the Majorana neutrino case, quarkonia are, however, composite systems characterized by several internal energy scales, which in turn may probe thermodynamical scales smaller than the temperature. Hence the situation is more complex than the one discussed in section 2. The energy scales characterizing a non-relativistic bound state are the typical momentum transfer in the bound state, which is of order $M v$, and the typical binding energy, which is of order $M v^{2}$. The parameter $v \ll 1$ is the relative heavy-quark velocity. This is of order $\alpha_{\mathrm{s}}$ for a Coulombic bound state. We call these scales the non-relativistic scales. The non-relativistic scales are hierarchically ordered: $M \gg M v \gg M v^{2}$. Effective field theories exploiting the nonrelativistic hierarchy in vacuum have been reviewed in [17]. For a weakly-coupled plasma, a relevant thermodynamical scale, which is smaller than the temperature, is the Debye mass, $m_{D}$, i.e. the inverse of the screening length of the chromoelectric interactions. This is of order $g T$, hence we have that parametrically $T \gg m_{D}$. We call the scales $T, m_{D}$ and possibly smaller scales the thermodynamical scales. For a discussion on the energy scale hierarchy in the case of $\Upsilon(1 S)$ produced in heavy-ion collisions at the LHC we refer to $[18,19]$. For first experimental evidence of suppression of excited bottomonium states at the LHC we refer to [20].
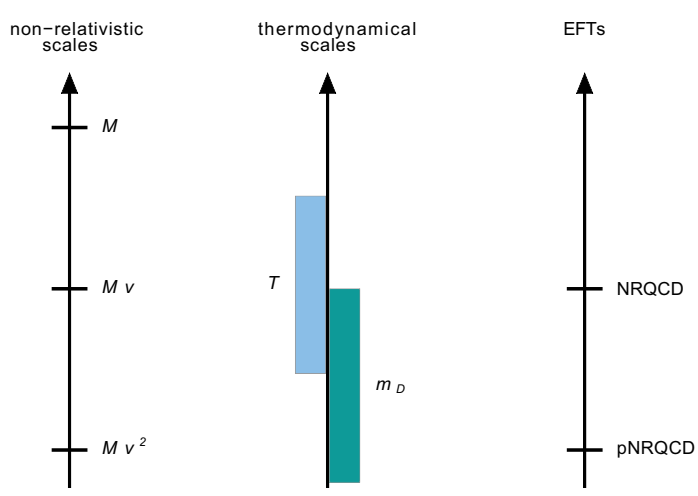

Figure 5. Scales and EFTs for quarkonium in a thermal bath.

The existence of a hierarchy of energy scales calls for a description of the system in terms of a hierarchy of EFTs of QCD, which is the fundamental theory in this case. Many EFTs are possible in dependence of the specific ordering of the thermodynamical scales with respect to the non-relativistic ones. These are schematically shown in figure 5. For temperatures larger than those considered in figure 5 quarkonium does not form. 
We call generically non-relativistic QCD (NRQCD) [21, 22] the EFT obtained from QCD by integrating out modes associated with the scale $M$ and possibly with thermodynamical scales larger than $M v$. The Lagrangian reads

$$
\mathcal{L}=\psi^{\dagger}\left(i D_{0}+\frac{\mathbf{D}^{2}}{2 M}+\ldots\right) \psi+\chi^{\dagger}\left(i D_{0}-\frac{\mathbf{D}^{2}}{2 M}+\ldots\right) \chi+\cdots+\mathcal{L}_{\text {light }},
$$

where $\psi(\chi)$ is here the field that annihilates (creates) the heavy (anti)quark.

We call generically potential NRQCD (pNRQCD) $[23,24]$ the EFT obtained from NRQCD by integrating out modes associated with the scale $M v$ and possibly with thermodynamical scales larger than $M v^{2}$. The degrees of freedom of pNRQCD are quark-antiquark states (cast conveniently in a colour singlet field $\mathrm{S}$ and a colour octet field $\mathrm{O}$ ), low energy gluons and light quarks propagating in the medium. The Lagrangian reads

$$
\begin{aligned}
\mathcal{L}=\int d^{3} r & \operatorname{Tr}\left\{\mathrm{S}^{\dagger}\left(i \partial_{0}-\frac{\mathbf{p}^{2}}{M}-V_{s}+\ldots\right) \mathrm{S}+\mathrm{O}^{\dagger}\left(i D_{0}-\frac{\mathbf{p}^{2}}{M}-V_{o}+\ldots\right) \mathrm{O}\right\} \\
+ & \operatorname{Tr}\left\{\mathrm{O}^{\dagger} \mathbf{r} \cdot g \mathbf{E} \mathrm{S}+\text { H.c. }\right\}+\frac{1}{2} \operatorname{Tr}\left\{\mathrm{O}^{\dagger} \mathbf{r} \cdot g \mathbf{E} \mathrm{O}+\text { c.c. }\right\}+\cdots+\mathcal{L}_{\text {light }},
\end{aligned}
$$

where $\mathbf{E}$ is the chromoelectric field and $g$ is now the $\mathrm{SU}(3)_{c}$ coupling. Both EFT Lagrangians (10) and (11) are of the form (1); only the field $\mathrm{S}$ is a gauge singlet. The pNRQCD Lagrangian is also organized as an expansion in $r: r$ is the distance between the heavy quark and antiquark, which is of order $1 /(M v)$. At leading order in $r$, the singlet field $\mathrm{S}$ satisfies a Schrödinger equation. Hence the Wilson coefficient $V_{s}$ may be interpreted as the singlet quarkonium potential. Similarly the Wilson coefficient $V_{o}$ may be interpreted as the octet quarkonium potential. The explicit expressions of the potentials depend on the explicit version of pNRQCD that is considered; in particular, if thermodynamical scales have been integrated out, $V_{S}$ and $V_{o}$ may depend on the temperature. We have set equal to one possible Wilson coefficients appearing in the second line of (11).

A key quantity for describing the expected quarkonium dilepton signal is the quarkonium dissociation width. At leading order it may be useful to distinguish between two dissociation mechanisms: gluodissociation, which is the dominant mechanism for $M v^{2} \gg m_{D}$, and dissociation by inelastic parton scattering, which is the dominant mechanism for $M v^{2} \ll m_{D}$. Beyond leading order the two mechanisms are intertwined and distinguishing between them would become arbitrary, whereas the physical quantity is the total decay width.

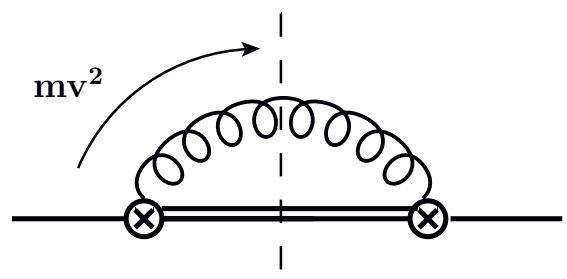

Figure 6. Quarkonium gluodissociation in pNRQCD.

Gluodissociation is the dissociation of quarkonium by absorption of a gluon from the medium [25, 26]. The gluon is lightlike or timelike (if it acquires an effective mass propagating through the medium). Gluodissociation is also known as singlet-to-octet break up [4, 27]. The process happens when the gluon has an energy of order $M v^{2}$. Hence gluodissociation can be described in pNRQCD. In particular, it can be calculated by cutting the gluon propagator in the pNRQCD diagram shown in figure 6 , where the single line stands for a quark-antiquark colour singlet propagator, the double line 
for a quark-antiquark colour octet propagator and the circle with a cross for a chromoelectric dipole vertex.

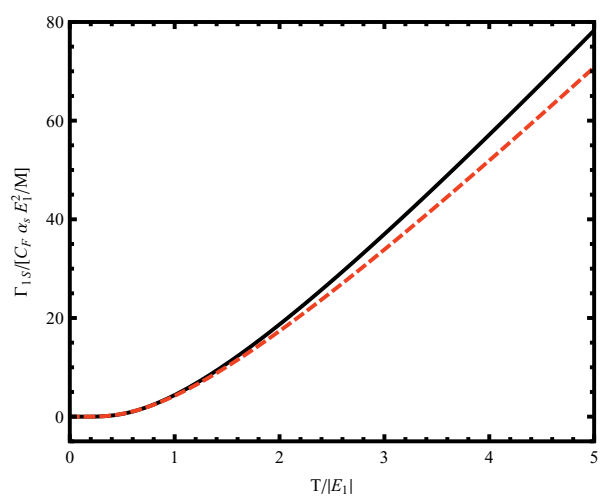

Figure 7. $\Gamma_{1 S}$ due to gluodissociation (continuous black line) vs the Bhanot-Peskin approximation (dashed red line).

Cutting rules at finite temperature [28-31] constrain the gluodissociation width of a quarkonium with quantum numbers $n$ and $l,(Q \bar{Q})_{n l}$, which is at rest with respect to the medium, to have the form

$$
\Gamma_{n l}=\int_{q_{\min }} \frac{d^{3} q}{(2 \pi)^{3}} n_{\mathrm{B}}(q) \sigma_{\text {gluo }}^{n l}(q),
$$

where $\sigma_{\text {gluo }}^{n l}$ is the in-vacuum cross section $(Q \bar{Q})_{n l}+$ gluon $\rightarrow Q+\bar{Q}$, and $n_{\mathrm{B}}$ is the Bose-Einstein distribution. The explicit leading order (LO) expression of $\sigma_{\text {gluo }}^{1 S}$ for a Coulombic $1 S$ state like the $\Upsilon(1 S)$ is $[27,32]$

$$
\sigma_{\text {gluo LO }}^{1 S}(q)=\frac{\alpha_{\mathrm{S}} C_{F}}{3} 2^{10} \pi^{2} \rho(\rho+2)^{2} \frac{E_{1}^{4}}{M q^{5}}\left(t(q)^{2}+\rho^{2}\right) \frac{\exp \left(\frac{4 \rho}{t(q)} \arctan (t(q))\right)}{e^{\frac{2 \pi \rho}{(q)}}-1},
$$

where $\rho=1 /\left(N_{c}^{2}-1\right), t(q)=\sqrt{q /\left|E_{1}\right|-1}, E_{1}=-M C_{F}^{2} \alpha_{\mathrm{s}}^{2} / 4$ and $C_{F}=\left(N_{c}^{2}-1\right) /\left(2 N_{c}\right)$. The corresponding width is shown in figure 7 . Note that the gluodissociation formula holds for temperatures such that $T \ll M v$ and $m_{D} \ll M v^{2} \sim\left|E_{1}\right|$. In figure 7 we also compare with a popular approximation, the so-called Bhanot-Peskin approximation $[33,34]$. This is the large $N_{c}$ limit of the full result (13) (but keeping $C_{F}=4 / 3$ in the overall normalization). Taking the large $N_{c}$ limit amounts at neglecting final state interactions, i.e. the rescattering of a $Q \bar{Q}$ pair in a colour octet configuration.
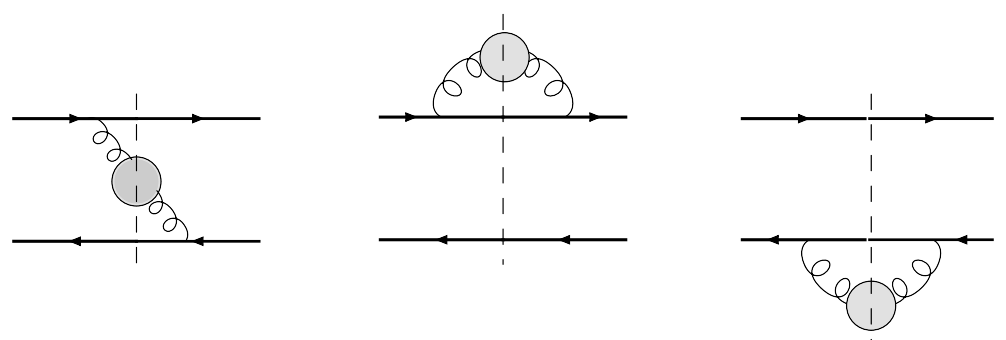

Figure 8. Quarkonium dissociation by inelastic parton scattering in NRQCD.

Dissociation by inelastic parton scattering is the dissociation of quarkonium by scattering with gluons and light-quarks in the medium [35,36]. The exchanged gluon is spacelike. Dissociation by inelastic parton scattering is also known as Landau damping [37]. Because external gluons are 
transverse, according to NRQCD each external gluon is suppressed by $T / M$, see (10). At leading order, we may therefore just consider contributions to the width coming from cutting diagrams with a self-energy insertion in one single gluon exchange [38]. If the exchanged gluon carries a momentum of order $M v$, then the relevant diagrams may be computed in NRQCD, see figure 8 . If the exchanged gluon carries a momentum much smaller than $M v$, then the relevant diagrams may be computed in pNRQCD, see figure 9. In both figures the dashed circle stands for a one-loop self-energy insertion.

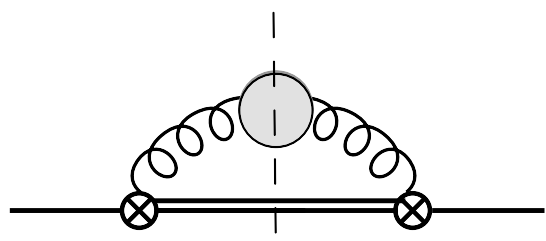

Figure 9. Quarkonium dissociation by inelastic parton scattering in $\mathrm{pNRQCD.}$

Cutting rules at finite temperature constrain the width by parton scattering of a quarkonium with quantum numbers $n$ and $l$, which is at rest with respect to the medium, to have the form

$$
\Gamma_{n l}=\sum_{p} \int_{q_{\min }} \frac{d^{3} q}{(2 \pi)^{3}} f_{p}(q)\left[1 \pm f_{p}(q)\right] \sigma_{p}^{n l}(q) .
$$

The sum runs over the different incoming and outgoing partons ( $p$ stands for parton, it may be either a light quark, $q$, for which the minus sign holds, or a gluon, $g$, for which the plus sign holds), with $f_{g}=n_{\mathrm{B}}$ and $f_{q}=n_{\mathrm{F}}\left(n_{F}\right.$ is the Fermi-Dirac distribution). The quantity $\sigma_{p}^{n l}$ is the in-medium cross section $(Q \bar{Q})_{n l}+p \rightarrow Q+\bar{Q}+p$. The convolution formula correctly accounts for Pauli blocking in the fermionic case (minus sign). Note that (14) differs from the corresponding gluodissociation formula (12) in the fact that it accounts for the thermal distributions of both the incoming and outgoing partons. Moreover, the cross section $\sigma_{p}^{n l}$ is not an in-vacuum cross section. Explicit expressions for the cross section in the case of a Coulombic $1 S$ state like the $\Upsilon(1 S)$ can be found in [38]. These are valid for temperatures such that $T \gg m_{D} \gg M v^{2} \sim\left|E_{1}\right|$. The corresponding width is shown in figure 10, where we have assumed three light quarks in the medium. Note the different normalization of the width with respect to figure 7.

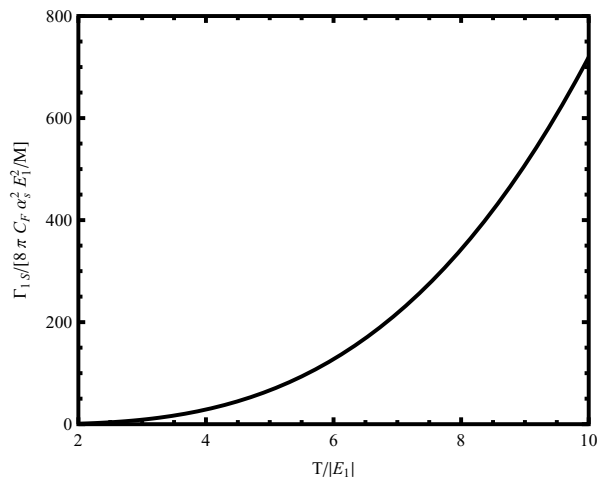

Figure 10. $\Gamma_{1 S}$ due to inelastic parton scattering. We have taken $m_{D} a_{0}=0.5$ and $\left|E_{1}\right| / m_{D}=0.5$, where $a_{0}$ is the Bohr radius. 


\section{Conclusions}

In a framework that makes close contact with modern effective field theories for non-relativistic particles at zero temperature, one can compute the thermal width of non-relativistic particles in a thermal bath in a systematic way. In the situation $M \gg T$ one may organize the computation in two steps and compute the physics at the scale $M$ as in vacuum. If other scales are larger than $T$, then also the physics of those scales may be computed as in vacuum. We have illustrated this on the examples of a heavy Majorana neutrino decaying in the early universe plasma and a heavy quarkonium dissociating in a weakly-coupled quark-gluon plasma.

\section{Acknowledgements}

I thank Simone Biondini, Nora Brambilla and Miguel Escobedo for collaboration on the work presented in section 2 and Nora Brambilla, Miguel Escobedo and Jacopo Ghiglieri for collaboration on the work presented in section 3. I acknowledge financial support from DFG and NSFC (CRC 110), and from the DFG cluster of excellence "Origin and structure of the universe" (www.universe-cluster.de).

\section{References}

[1] N. Isgur and M. B. Wise, Phys. Lett. B 232, 113 (1989).

[2] E. Eichten and B. R. Hill, Phys. Lett. B 234, 511 (1990).

[3] M. Le Bellac, Thermal Field Theory (Cambridge University Press, Cambridge, 1996) 272 p.

[4] N. Brambilla, J. Ghiglieri, A. Vairo and P. Petreczky, Phys. Rev. D 78, 014017 (2008) [arXiv:0804.0993 [hep-ph]].

[5] P. Minkowski, Phys. Lett. B 67, 421 (1977).

[6] M. Gell-Mann, P. Ramond and R. Slansky, Conf. Proc. C 790927, 315 (1979) [arXiv:1306.4669 [hep-th]].

[7] M. Fukugita and T. Yanagida, Phys. Lett. B 174, 45 (1986).

[8] M. A. Luty, Phys. Rev. D 45, 455 (1992).

[9] M. Drewes, Int. J. Mod. Phys. E 22, 1330019 (2013) [arXiv:1303.6912 [hep-ph]].

[10] A. D. Sakharov, Pisma Zh. Eksp. Teor. Fiz. 5, 32 (1967) [JETP Lett. 5, 24 (1967)] [Sov. Phys. Usp. 34, 392 (1991)] [Usp. Fiz. Nauk 161, 61 (1991)].

[11] S. Biondini, N. Brambilla, M. A. Escobedo and A. Vairo, JHEP 1312, 028 (2013) [arXiv:1307.7680 [hep-ph]].

[12] K. Kopp and T. Okui Phys. Rev. D 84, 093007 (2011) [arXiv:1108.2702 [hep-ph]].

[13] A. Salvio, P. Lodone and A. Strumia, JHEP 1108, 116 (2011) [arXiv:1106.2814 [hep-ph]].

[14] M. Laine and Y. Schröder, JHEP 1202, 068 (2012) [arXiv:1112.1205 [hep-ph]].

[15] D. Bodeker and M. Wormann, arXiv:1311.2593 [hep-ph].

[16] T. Matsui and H. Satz, Phys. Lett. B 178, 416 (1986).

[17] N. Brambilla, A. Pineda, J. Soto and A. Vairo, Rev. Mod. Phys. 77, 1423 (2005) [hep$\mathrm{ph} / 0410047]$.

[18] N. Brambilla, M. A. Escobedo, J. Ghiglieri, J. Soto and A. Vairo, JHEP 1009, 038 (2010) [arXiv:1007.4156 [hep-ph]].

[19] A. Vairo, AIP Conf. Proc. 1317, 241 (2011) [arXiv:1009.6137 [hep-ph]].

[20] S. Chatrchyan et al. [CMS Collaboration], Phys. Rev. Lett. 107, 052302 (2011) [arXiv:1105.4894 [nucl-ex]]. 
[21] W. E. Caswell and G. P. Lepage, Phys. Lett. B 167, 437 (1986).

[22] G. T. Bodwin, E. Braaten and G. P. Lepage, Phys. Rev. D 51, 1125 (1995) [Erratum-ibid. D 55, 5853 (1997)] [hep-ph/9407339].

[23] A. Pineda and J. Soto, Nucl. Phys. Proc. Suppl. 64, 428 (1998) [hep-ph/9707481].

[24] N. Brambilla, A. Pineda, J. Soto and A. Vairo, Nucl. Phys. B 566, 275 (2000) [hep-ph/9907240].

[25] D. Kharzeev and H. Satz, Phys. Lett. B 334, 155 (1994) [arXiv:hep-ph/9405414].

[26] X. M. Xu, D. Kharzeev, H. Satz and X. N. Wang, Phys. Rev. C 53, 3051 (1996) [arXiv:hep$\mathrm{ph} / 9511331]$.

[27] N. Brambilla, M. A. Escobedo, J. Ghiglieri and A. Vairo, JHEP 1112, 116 (2011) [arXiv:1109.5826 [hep-ph]].

[28] R. L. Kobes and G. W. Semenoff, Nucl. Phys. B 260, 714 (1985).

[29] R. L. Kobes and G. W. Semenoff, Nucl. Phys. B 272, 329 (1986).

[30] P. F. Bedaque, A. K. Das and S. Naik, Mod. Phys. Lett. A 12, 2481 (1997) [hep-ph/9603325].

[31] F. Gelis, Nucl. Phys. B 508, 483 (1997) [hep-ph/9701410].

[32] F. Brezinski and G. Wolschin, Phys. Lett. B 707, 534 (2012) [arXiv:1109.0211 [hep-ph]].

[33] M. E. Peskin, Nucl. Phys. B 156, 365 (1979).

[34] G. Bhanot and M. E. Peskin, Nucl. Phys. B 156, 391 (1979).

[35] L. Grandchamp and R. Rapp, Phys. Lett. B 523, 60 (2001) [hep-ph/0103124].

[36] L. Grandchamp and R. Rapp, Nucl. Phys. A 709, 415 (2002) [hep-ph/0205305].

[37] M. Laine, O. Philipsen, P. Romatschke and M. Tassler, JHEP 0703, 054 (2007) [arXiv:hep$\mathrm{ph} / 0611300]$.

[38] N. Brambilla, M. A. Escobedo, J. Ghiglieri and A. Vairo, JHEP 1305, 130 (2013) [arXiv:1303.6097 [hep-ph]]. 